\title{
A chemical analysis of five hot stars towards the Galactic centre
}

\author{
K. E. Munn ${ }^{1}$, P. L. Dufton ${ }^{1}$, S. J. Smartt ${ }^{2}$, and N. C. Hambly ${ }^{3}$ \\ 1 Department of Pure \& Applied Physics, The Queen's University of Belfast, BT7 1NN, Northern Ireland, UK \\ 2 Institute for Astronomy, University of Cambridge, Madingley Road, Cambridge CB3 OBA,UK \\ ${ }^{3}$ Institute for Astronomy, University of Edinburgh, Blackford Hill, Edinburgh EH9 3HJ, Scotland, UK
}

Received 21 October 2003 / Accepted 15 February 2004

\begin{abstract}
High resolution echelle spectroscopy is presented for thirteen stars lying in the direction of the Galactic centre which, on the basis of photographic photometry and low dispersion spectroscopy, have been classified as early-B-type. Eight of these stars have large rotational velocities which preclude a detailed analysis. The five stars with moderate to low projected rotational velocities have been analysed using model atmosphere techniques to determine atmospheric parameters and chemical compositions. Two of these stars appear to be evolved blue horizontal branch objects on the basis of their chemical compositions and small projected rotational velocity. The evolutionary status of a third is ambiguous but it is probably a post-asymptotic-giant branch star. The remaining two objects are probably young massive stars and show enhanced abundances of $\mathrm{N}, \mathrm{C}, \mathrm{Mg}$ and $\mathrm{Si}$, consistent with their formation in the inner part of the Galactic disk. However their $\mathrm{O}$ abundances are normal, confirming results found previously for other early-type stars, which would imply a flat abundance gradient for this element in the inner region of our Galaxy.
\end{abstract}

Key words. stars: early-type - stars: atmospheres - stars: abundances - Galaxy: evolution - Galaxy: center Galaxy: abundances

\section{Introduction}

Massive, early-type stars can be used to investigate the chemical composition and recent evolutionary history of galaxies (see, for example, the proceedings of the second BoulderMunich workshop - Howarth 1998). Their large luminosities allow even main sequence stars to be observed at high spectral resolution and signal-to-noise $(S / N)$ ratio in distant regions of our galaxy (Dufton 1998; Smartt et al. 1996b; Gummersbach et al. 1998), and in nearby galaxies such as the Magellanic System (Rolleston et al. 1996, 2003; Korn et al. 2002). Additionally early-type supergiants (with luminosities up to $10^{6}$ that of the Sun) can now be observed in galaxies such as M 31 and M 33 (Trundle et al. 2002; Monteverde et al. 1997; Smartt et al. 2001; Bresolin et al. 2002) and other local group irregular galaxies (see, for example, Venn et al. 2001). Their short evolutionary lifetimes then ensure that they provide information on the current status of the host galaxy.

The atmospheres of B-type main-sequence stars have been reliably modelled to yield accurate atmospheric parameters (see, for example, Gies \& Lambert 1992; Kilian 1992). Additionally when differential methods are adopted, it may be possible to deduce relative abundances to an accuracy of 0.1 dex (see for example Smartt et al. 1996b). Abundance estimates are also less subject to the uncertainties implicit in other

Send offprint requests to: P. L. Dufton,

e-mail: p.dufton@qub.ac.uk methods, such as dust affecting abundances from emission line plasmas (e.g. H II regions), or contamination from mixing with nuclear material processed from the stellar interior for highly evolved objects (e.g. Planetary Nebulae). However although these objects are relatively unevolved, there is evidence that in some cases their atmosphere may be contaminated by rotational mixing (Gies \& Lambert 1992; Mathys et al. 2002).

Previous stellar investigations of the chemical composition of the Galactic disk have concentrated mainly on the anticentre direction (see, for example, Smartt et al. 1996a,b; Gummersbach et al. 1998). More recently stellar estimates of large scale abundance gradients (Smartt \& Rolleston 1997; Rolleston et al. 2000; Daflon \& Cunha 2002) have become available. By contrast, studies towards the Galactic centre have been hampered by the large extinction at optical wavelengths. However, preliminary investigations of stars with galactic latitudes $2^{\circ} \leq|b| \leq 10^{\circ}$ have shown that it is possible to map both the inner region of the galaxy (Smartt et al. 2001) and even beyond the Galactic centre (Ryans et al. 1997). These last two papers and, for example, Venn et al. (1998) used the catalogues of southern luminous stars by Reed (1993) and Reed \& Beatty (1995) to search for early-type candidates. The target selection was based on colour-colour and colour-magnitude criteria and these catalogues have a faint visual magnitude cut-off of $\sim 12.5$ and may not be complete.

Hence we believe that the available catalogues for these Galactic directions are not ideal for selecting blue stars within 
the inner few kiloparsecs of the disk. In order to further these studies, we have attempted to identify more potential targets from a wide-field survey technique employing both multi-colour photometry and preliminary low-resolution spectroscopy for spectral-type classification. The former was obtained from UK photographic Schmidt plates and films (including new exposures specially obtained for this project), while the latter used the FLAIR multi-object spectrograph on the UK Schmidt telescope. Ten Schmidt fields towards the Galactic Centre were surveyed (Dufton et al. 1999, 2001) and spectroscopy obtained for approximately 600 blue targets. Of these, 56 stars have spectra consistent with a B-type spectral classification. Here we report on high dispersion echelle spectroscopy of a subset of these targets.

\section{Observations and data reduction}

High dispersion spectroscopy was obtained for thirteen targets using the UCLES spectrograph on the Anglo-Australian Telescopes on the nights of the 11 and 12 July 2000. Targets were selected from the list of blue objects discussed by Dufton et al. (1999, 2001), which from low dispersion FLAIR spectroscopy appeared to be B-type stars lying towards the Galactic Centre. High priority was given to those targets which appeared to be early-B type and whose magnitudes implied that if they were young stars, then they would be situated at distances of $3-8 \mathrm{kpc}$ from the Sun in the direction of the Galactic Centre.

The 31 lines $\mathrm{mm}^{-1}$ grating was used together with a Tek CCD, which provided complete wavelength coverage from approximately 3900 to $4900 \AA$ at a resolution of approximately $0.1 \AA$ in two separate settings. Additional coverage from approximately 5400 to $7400 \AA$ was obtained for three of the targets. Bias and flat field frames were obtained at the beginning and end of each night while Th-Ar wavelength calibration frames were interleaved with the stellar spectra.

The two dimensional CCD frames were reduced using standard procedures within IRAF (Tody 1986). Preliminary processing of the CCD frames, such as overscan correction and flatfielding was performed using the CCDRED package (Massey 1997), while cosmic-ray removal, stellar and sky spectrum extraction and wavelength calibration were undertaken using DOECSLIT (Willmarth \& Barnes 1994).

After co-addition of the extracted spectra, further analysis was carried out using the STARLINK DIPSO package (Howarth et al. 1994). Such reductions included normalisation of the spectra, estimating the stellar radial and rotational velocities and measuring the absorption line strengths. Projected rotational velocities $(v \sin i)$ were estimated by comparing observed profiles with theoretical profiles that had been convolved with a rotational broadening function. This method is effectively identical to that discussed by Lennon et al. (1991). For two stars, the lines showed no significant broadening and only upper limits could be placed on their projected rotational velocities. The stellar radial velocities and metal line equivalent widths were estimated from non-linear-least-squares fitting using Gaussian profiles for the absorption lines and a low order polynomial to represent the continuum. Full details of these methods can be found in Rolleston et al. (1999) and references therein. The

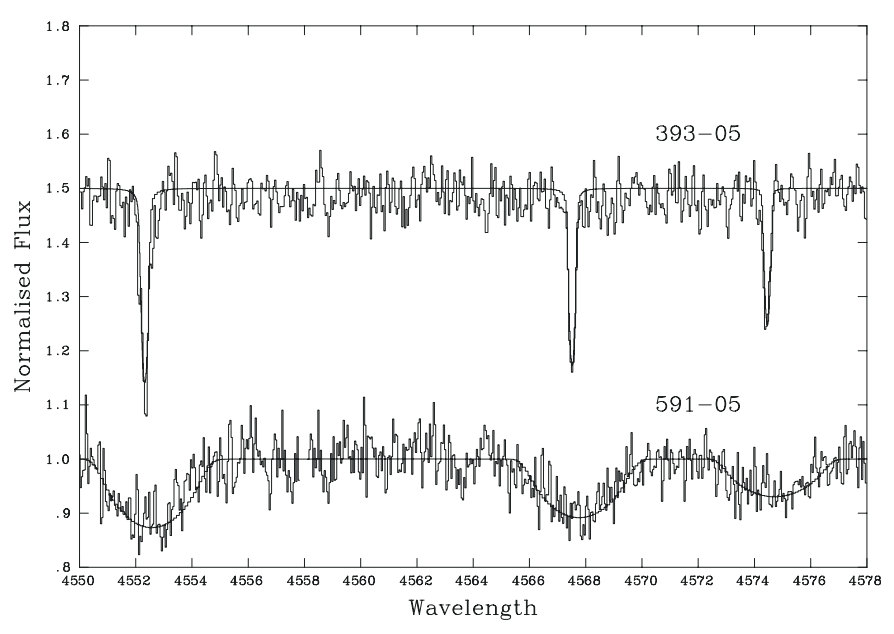

Fig. 1. Spectrum for the stars 393-03 and 591-05 covering the Si III triplet near $4560 \AA$ A. Also shown are synthetic spectra for the adopted atmospheric parameters and silicon abundances. The theoretical spectrum for 591-05 has been convolved with a rotational broadening function to take account of its significant projected rotational velocity.

adoption of Gaussian profiles particularly for stars with significant projected rotational velocities may not be appropriate. For these cases, we undertook tests using rotational broadened profiles in the fitting procedures (as discussed in Lennon et al. 1991). Differences in the equivalent width estimates were typically ten percent or less, implying that the use of Gaussian profiles was acceptable.

Observational details are summarised in Table 1 and includes stellar co-ordinates, radial velocities (transformed to a heliocentric frame) and projected rotational velocities, together with typical $S / N$ ratios for the co-added spectra and total exposure times. Note that as the observing conditions varied significantly during the two nights, the exposure times should be treated with caution. $U B V$ measurements for five stars were obtained using the $1.0 \mathrm{~m}$ telescope at the SAAO (Kilkenny, private communication). For four of the stars multiple observations were carried out with the visual magnitudes and colours agreeing to within $0.01 \mathrm{mag}$, the exception being 456-39 where only one observation was obtained. For the remaining stars photographic magnitudes were taken from Dufton et al. (1999, 2001). The equivalent widths estimates, which are too extensive to publish here, are available from the authors on request.

The quality of the spectra varied significantly as can be seen from the sample spectra in Fig. 1 for the stars 393-03, which has narrow metallic absorption lines and for star 591-05, which has the largest projected rotational velocity of the stars that were analysed. This latter star represents the lower threshold of the spectra which were considered suitable for further analysis. Of the fourteen stars listed in Table 1, five targets (marked by an asterisk) were considered suitable for further investigation on the basis of their spectral types, relatively low projected rotational velocities and $S / N$ ratios.

Differential methods have been adopted in the model atmosphere analyses discussed below, with our targets being analysed relative to bright B-type stars with similar atmospheric parameters. The observational data for the standards were 
Table 1. Observational details for targets observed with the Anglo Australian Telescope. The five stars that have been included in the model atmosphere analysis are marked with an asterisk. Photographic $V$ magnitudes are quoted to one decimal place reflecting likely errors of at least 0.1 mag. Total exposure times $\left(T_{\exp }\right)$ are given in minutes.

\begin{tabular}{|c|c|c|c|c|c|c|c|c|c|c|c|c|}
\hline \multirow[t]{2}{*}{ Field } & \multirow[t]{2}{*}{ Star } & \multirow[t]{2}{*}{ RA (2000) } & \multirow[t]{2}{*}{ Dec } & \multirow[t]{2}{*}{$V$} & \multirow[t]{2}{*}{$(B-V)$} & \multirow[t]{2}{*}{$(U-B)$} & \multirow{2}{*}{$\begin{array}{r}V_{\text {rad }} \\
\mathrm{km} \mathrm{s}^{-1}\end{array}$} & \multirow{2}{*}{$\begin{array}{r}v \sin i \\
\mathrm{~km} \mathrm{~s}^{-1}\end{array}$} & \multicolumn{2}{|c|}{ Blue } & \multicolumn{2}{|c|}{ Red } \\
\hline & & & & & & & & & $S / N$ & $T_{\exp }$ & $S / N$ & $T_{\text {exp }}$ \\
\hline 279 & $8^{*}$ & 18133.90 & -453156.4 & 12.48 & -0.127 & -0.719 & 42 & $\leq 10$ & 49 & 40 & 21 & 15 \\
\hline 279 & 9 & 174314.69 & -45232.8 & 13.7 & - & - & - & 90 & 26 & 105 & - & - \\
\hline 279 & $19 *$ & 17392.20 & -45038.7 & 13.05 & 0.214 & -0.640 & -55 & 30 & 26 & 75 & 20 & 15 \\
\hline 391 & 9 & 165450.83 & -341610.3 & 13.5 & - & - & - & 16 & 25 & 90 & - & - \\
\hline 391 & 23 & 165918.33 & -332535.2 & 12.0 & - & - & - & 160 & 25 & 41 & - & - \\
\hline 393 & $3 *$ & 174959.91 & -353240.9 & 12.94 & 0.045 & -0.712 & -26 & $\leq 10$ & 31 & 52 & - & - \\
\hline 393 & 32 & 174858.41 & -35 1835.0 & 12.7 & - & - & - & 120 & 17 & 15 & - & - \\
\hline 454 & 10 & 171738.60 & -284056.7 & 12.2 & - & - & - & 190 & 35 & 20 & - & - \\
\hline 454 & 27 & 171425.59 & $-31 \quad 1216.6$ & 13.8 & - & - & - & 110 & 13 & 30 & - & - \\
\hline 456 & $39 *$ & 18527.05 & -28183.7 & 12.00 & 0.323 & -0.576 & -68 & 45 & 51 & 134 & 26 & 15 \\
\hline 589 & 33 & 174911.23 & -192950.3 & 12.3 & - & - & - & 140 & 19 & 17 & - & - \\
\hline 591 & $5^{*}$ & 183935.58 & -191410.8 & 13.24 & 0.246 & -0.638 & -26 & 130 & 26 & 60 & - & - \\
\hline 591 & 30 & 183916.88 & -18118.0 & 13.2 & - & - & - & 120 & 19 & 60 & - & - \\
\hline
\end{tabular}

obtained using the $0.9 \mathrm{~m}$ Coudé feed telescope at the Kitt Peak national Observatory for HR 1765, HR 5595 and HR 2387 or from the $2.1 \mathrm{~m}$ and $2.7 \mathrm{~m}$ telescopes at the McDonald Observatory for $\mathrm{BD}+56^{\circ} 502$. These observations, which had $S / N$ ratios in excess of 200 , were reduced using similar methods to those described above and further details can be found in Hambly et al. (1997) and Vrancken et al. (2000) respectively.

\section{Method of analysis}

A Local Thermodynamic Equilibrium (LTE) analysis of all five stars was carried out using theoretical models based on the grid of line-blanketed model atmospheres from the ATLAS9 code of Kurucz (1991) and LTE radiative transfer codes. The determination of the atmospheric parameters is an iterative process requiring consistent estimates for the effective temperature, gravity, microturbulence and metallicity. Below we discuss the criteria that were used for estimating each parameter.

\subsection{Atmospheric parameters}

\subsubsection{Effective temperature}

An estimate of the effective temperature, $T_{\text {eff }}$, of four of the five stars was made by balancing the abundance estimates for silicon from different ionisation stages (viz. SiII/SiIII or SiIII/SiIV), adopting a preliminary estimate for the surface gravity and a microturbulent velocity of $\xi=5 \mathrm{~km} \mathrm{~s}^{-1}$. Although the latter was subsequently found to be too small for some of the stars (see Sect. 3.1.3), test calculations showed that the effective temperature estimates were not significantly affected when these larger values were adopted. In the spectra of the star 591-05, it was only possible to reliably identify lines from one ionisation stage for silicon and hence the effective temperature was estimated from the neutral helium lines assuming that the helium abundance was normal. Profile fittings of the very weak HeII line at $4685 \AA$ implied an upper limit to the effective temperature of this star is $\sim 27000 \mathrm{~K}$, in agreement with our estimate from the He I spectrum.

Abundance analyses showed that four of our target stars appeared to have enhanced metallicity. In these cases, effective temperatures (and other atmospheric parameters) were also estimated adopting atmospheres with enhanced (+0.5 dex) metallicities. For consistency, the same methods (with normal metallicity atmospheres) were employed in estimating the temperatures of the standard stars. Table 2 summarises all these estimates. Uncertainties in these effective temperature estimates will arise from several sources, viz. the moderate $S / N$ ratio and resolution of the spectra, the values assumed for other atmospheric parameters, and the assumptions (e.g. LTE) made in the model atmosphere analyses. We believe that our equivalent width measurements for our target stars should normally be accurate to $20 \%$. Taking the worst case scenario, of errors of this magnitude but with different signs in the Si III and Si IV would translate into an error in the effective temperature of approximately $2000 \mathrm{~K}$. The observational data for the standard stars is of higher quality and the corresponding errors will be less than $1000 \mathrm{~K}$. In the case of errors in the adopted gravity and microturbulence (as discussed below), the corresponding error in the effective temperature would be relatively small (typically less than $1000 \mathrm{~K}$ ). Finally possible errors due to assumptions made in the model atmosphere calculations are difficult to estimate. In the case of our assumption of LTE, non-LTE results that we are currently calculating using the TLUSTY and SYNSPEC codes (Hubeny 1988; Hubeny \& Lanz 1988) indicate that for the Si III/Si IV ionization equilibrium the uncertainty is less than $1000 \mathrm{~K}$. Hence the uncertainty in the effective temperature estimates for our targets is probably dominated by the quality of the observational data and is of the order of $2000 \mathrm{~K}$. 
Table 2. Adopted atmospheric parameters for the five galactic centre targets. Where appropriate estimates are listed for both normal and enhanced (+0.5 dex) metallicity model atmospheres. The atmospheric parameters of the standard stars deduced using normal metallicity atmospheres are also shown.

\begin{tabular}{|c|c|c|c|c|c|c|}
\hline \multirow[t]{2}{*}{ Star } & \multicolumn{2}{|c|}{ Temp. $(\mathrm{K})$} & \multicolumn{2}{|c|}{$\log g$} & \multicolumn{2}{|r|}{$\xi$} \\
\hline & Normal & Enhanced & Normal & Enhanced & Normal & Enhanced \\
\hline $393-03$ & 23000 & 22000 & 4.5 & 4.5 & 5 & 5 \\
\hline HR 1765 & 23500 & & 4.0 & & 6 & \\
\hline 279-08 & 20000 & 20000 & 4.5 & 4.5 & 5 & 5 \\
\hline HR 5595 & 19000 & & 4.4 & & 5 & \\
\hline $456-39$ & 26000 & 25000 & 4.2 & 4.1 & 10 & 10 \\
\hline HR 2387 & 26000 & & 3.8 & & 10 & \\
\hline 279-19 & 24000 & & 3.3 & & 15 & \\
\hline $\mathrm{BD}+56^{\circ} 502$ & 23500 & & 3.5 & & 13 & \\
\hline 591-05 & 27000 & 27000 & 3.8 & 3.8 & 15 & 15 \\
\hline HR 2387 & 26000 & & 3.8 & & 10 & \\
\hline
\end{tabular}

\subsubsection{Gravity}

Gravities were estimated by comparing observed profiles for the hydrogen Balmer lines with theoretical profiles. Normalisation of the observed spectra was complicated by the relatively large widths of these lines, which made the continuum placement difficult. This difficulty was ameliorated by using adjacent orders to determine an appropriate blaze profile. This was then used to rectify the spectrum prior to normalisation. For the blue spectra the $\mathrm{H} \beta, \mathrm{H} \gamma, \mathrm{H} \delta$ and $\mathrm{H} \epsilon$ lines were considered and where available a comparison was also made with the $\mathrm{H} \alpha$ line.

The final adopted gravities are listed in Table 2 for both normal and enhanced metallicities. Also listed are values for the standard stars for normal metallicity models, which were estimated in a similar manner. The uncertainties in these estimates will arise from similar sources as for the effective temperature estimates. The quality of the observed spectra for our targets, implies a fitting uncertainty of approximately 0.2 dex, whilst our estimated errors in the effective temperature estimates would introduce a similar uncertainty. For our higher gravity targets, non-LTE effects should small and of the order of 0.1 dex for the low gravity object 279-19 (see, for example, Mihalas 1972).

\subsubsection{Microturbulent velocity}

As was discussed previously the microturbulent velocity, $\xi$, was initially assumed to be $5 \mathrm{~km} \mathrm{~s}^{-1}$. A curve-of-growth analysis revealed that, although this value was appropriate for the stars 393-03 and 279-08, it was too low for the three remaining targets and also for some of the standards. Indeed, plots of the derived abundance against observed line strength for ions such as NII and OII revealed a positive sloping gradient. The microturbulent velocity for each star was then varied until elemental abundances were found to be independent of line strength. The adopted values of $\xi$ are again listed in Table 2. It should be noted that the microturbulent velocity assumed for star 591-05 $\left(\xi=15 \mathrm{~km} \mathrm{~s}^{-1}\right)$ is effectively a lower limit as most of the absorption features are on or near to the linear part of the curve of growth. The cause of this particularly high microturbulent velocity in a star with atmospheric parameters appropriate a near main sequence evolutionary state is unclear. However, adopting a larger estimate would alter the derived abundance estimates by typically less than $0.1 \mathrm{dex}$. For the other stars, the major uncertainty in the determination of the microturbulent velocity will be errors in the equivalent width measurements; these will translate into uncertainties of $\pm 5 \mathrm{~km} \mathrm{~s}^{-1}$ or more in this quantity.

\subsection{Abundance analysis}

Using the atmospheric parameters discussed above, the metal lines in our targets were used to estimate absolute abundances using atomic data taken mainly from Jeffery (1991). These are listed in Table 3, together with sample standard deviations. They are based on a logarithmic scale relative to hydrogen where the hydrogen abundance is 12.0 dex. As discussed above, an initial abundance analysis revealed a metal enhancement of $\sim 0.5$ dex for all of the stars except 279-19. Consequently for these four stars the values listed in Table 3 are based on the atmospheric parameters in Table 2 appropriate to an enhanced metallicity of $+0.5 \mathrm{dex}$. However test calculations indicated that in most cases use of the atmospheric parameters and models with a normal metallicity would have changed these absolute abundance estimates by less than 0.2 dex.

Uncertainties in the absolute and differential abundances will arise from the same sources as those discussed for the atmospheric parameters (see, for example, Sect. 3.1.1). An observational error of $20 \%$ translates into an abundance error 
ranging from approximately $0.1 \mathrm{dex}$ for weak features to 0.3 dex for lines on the flat part of the curve of growth. As such errors should be randomly distributed, the error in the mean abundances for species with a rich spectrum should be smaller. The uncertainties due to possible errors in the atmospheric parameters will vary with ionic species and will also depend on the stellar atmospheric parameters. Test calculations indicate that the major errors arise from the uncertainties in effective temperature and microturbulence and can range upto 0.4 dex. Note that these errors would be systematic for any given ionic species (although in the case of the microturbulence would depend on the strength of the line). Non-LTE effects will also depend on the ion species and the adopted atmospheric parameters. Test calculations using the grid discussed in Sect. 3.1.1 indicate that for most species the effects are relatively small and result in abundance changes of the order of 0.2 dex, although for the hotter targets the effects become larger. For the differential abundances, the errors due the observational uncertainties will effectively remain unchanged. Those due to the choice of the atmospheric parameters will be reduced when the errors in the atmospheric parameters are systematic. Additionally errors due to non-LTE effects should be significantly reduced provided the target and standard have similar atmospheric parameters.

Line-by-line differential abundance analyses were also undertaken for all the target stars using both normal and, where appropriate, enhanced metallicity atmospheric parameters and model atmospheres. The parameters for the standard stars are those listed in Table 2 for normal metallicities. Unfortunately the spectral coverage of the standard stars did not include all of the lines observed in the target stars and so the differential analyses was limited to the common subsets of spectral lines. The results are shown in Tables 4 to 7, where the stars have been grouped together on the basis of their inferred evolutionary status (see Sect. 4). These differential abundance estimates should be less susceptible to systematic errors due to uncertainties in the atomic data and also any non-LTE effects. Further details on the methods used can be found in, for example, Rolleston et al. (1999).

\section{Results and discussion}

As Tables 3 to 7 demonstrate, the chemical compositions of our target stars are not homogeneous. Hence it is appropriate to divide the stars into subgroups. Below, the results are discussed firstly for two narrow-lined stars (393-03 and 279-08), followed by two broad-lined stars (456-39 and 591-05) and finally the lower gravity star (279-19). For the first four targets stars, the differential abundances estimates discussed below will normally be for the enhanced metallicity models, although the principle conclusions would be unaffected if the results for the normal metallicity model atmospheres were adopted.

All five targets are plotted in an effective temperature versus gravity diagram in Fig. 2. Also shown are the theoretical positions of the zero-age hydrogen burning main- sequence, zero-age horizontal branch, post-horizontal branch and post-asymptotic giant branch (post-AGB), taken from
Bertelli et al. (1994), Sweigart (1987), Dorman et al. (1993) and Schönberner (1993) respectively.

\subsection{Narrow-lined stars $-279-08$ and 393-03}

The stars 279-08 and 393-03 have been grouped together as both have very sharp-lined spectra with line widths compatible with a zero projected rotational velocity. Additionally they have similar atmospheric parameters (see Table 2) and chemical compositions (see Tables 3 and 4), with for example significant enhancements for carbon and nitrogen.

The absolute helium abundance in both stars appears to be relatively normal with that for 279-08 being slightly smaller (see Table 3). It is hence surprising that whilst 393-02 has a near zero differential abundance that for 279-08 implies a helium enhancement of near a factor of two (see Table 4). HR5595 was the standard star selected for the differential analysis of this latter target and due to the respective wavelength coverage, only four non-diffuse helium lines could be included in this analysis. Hence, the significance of this apparent helium enrichment is uncertain (particularly in the light of the absolute helium abundances) and may reflect a low helium abundance estimate in the standard or errors in the adopted atmospheric parameters.

The enhancements of carbon, nitrogen and oxygen in the two target stars are very similar. The largest enhancement is for nitrogen (0.9-1.0 dex), followed by carbon (0.6-0.7 dex) and oxygen with $(0.3$ dex $)$. The differential abundances of the heavier metals are also very similar with magnesium having a near normal composition and other elements typical enhancements of 0.3 dex.

This abundance pattern shows some similarities to that found previously by Trundle et al. (2001) for a field star HD 135485. This is illustrated in Table 4, where the differential abundances for our two targets are compared with those of HD 135485 (using the standard star HR 5595). These results are taken directly from Trundle et al. with the generally lower standard deviations being at least in part due to the higher $S / N$ ratio (of the order of 200) of their spectra. From an inspection of this table, it would appear that the CNO overabundances are similar in our two target stars and in HD 135485. Additionally the helium enhancements in 279-08 and HD 135485 are similar - however given the uncertainties of our helium abundances estimates this may be coincidental. The abundances of the heavier elements for our targets and HD 135485 are also in reasonable agreement, although the $\mathrm{Mg}$ enhancement observed in HD 135485 is not observed in our targets.

Trundle et al. believed that the most plausible explanation for the chemical composition of HD 135485 was that it was an evolved star that had formed from material with an enhanced metallicity. Subsequently its atmospheric composition had been modified by diffusion and the mixing of nucleosynthetically processed material from its interior. It is possible that a modified version of this hypothesis that takes account of the normal $\mathrm{Mg}$ abundance may be appropriate to our targets.

Behr et al. (1999) have studied high resolution optical spectra of 13 blue horizontal branch stars in the globular cluster 
Table 3. Absolute elemental abundance estimates for all five stars, together with sample standard deviations. The first column lists the ionization stages that were used to estimate the element abundances. Also included are the absolute elemental abundances of $\gamma$ Peg, an apparently chemically normal star lying within the solar neighbourhood.

\begin{tabular}{lllllll}
\hline \hline Ion & \multicolumn{5}{c}{ Logarithmic absolute abundances: $\log \epsilon(X)=\log \frac{X}{\mathrm{H}}+12$} \\
& $\gamma$ Peg & $279-08$ & $393-03$ & $456-39$ & $591-05$ & $279-19$ \\
\hline He I & - & $10.77 \pm 0.21$ & $10.95 \pm 0.22$ & $10.98 \pm 0.28$ & $10.70 \pm 0.12$ & $10.90 \pm 0.30$ \\
C II & $8.22 \pm 0.31$ & $8.58 \pm 0.37$ & $8.71 \pm 0.32$ & $8.21 \pm 0.29$ & $8.71 \pm 0.56$ & $7.83 \pm 0.87$ \\
N II & $7.79 \pm 0.24$ & $8.25 \pm 0.15$ & $8.56 \pm 0.19$ & $8.12 \pm 0.17$ & $8.19 \pm 0.26$ & $7.57 \pm 0.41$ \\
O II & $8.72 \pm 0.32$ & $8.39 \pm 0.29$ & $8.47 \pm 0.20$ & $8.69 \pm 0.16$ & $8.69 \pm 0.20$ & $8.60 \pm 0.20$ \\
Mg II & 7.22 & 7.48 & 7.69 & 7.42 & 7.46 & 6.98 \\
Al III & $6.14 \pm 0.05$ & $6.21 \pm 0.16$ & $6.61 \pm 0.25$ & $6.20 \pm 0.01$ & - & - \\
Si II/III & $6.90 \pm 0.15$ & $7.16 \pm 0.29$ & $7.39 \pm 0.17$ & $7.44 \pm 0.17$ & $7.53 \pm 0.11$ & $7.07 \pm 0.06$ \\
P II & - & $\leq 5.3$ & $\leq 5.5$ & - & - & - \\
S II & $7.01 \pm 0.31$ & $7.39 \pm 0.31$ & $7.65 \pm 0.26$ & - & - & - \\
Cr II & - & $\leq 6.4$ & $\leq 7.0$ & - & - & - \\
Fe III & $6.39 \pm 0.82$ & - & $7.58 \pm 0.06$ & $7.80 \pm 0.37$ & - & 7.56 \\
\hline
\end{tabular}

Table 4. Differential abundance analyses for the stars 279-08 and 393-03. For each star there are four columns, listing the number of lines included, differential abundances (using the standards listed in Table 2) adopting either normal or metal enhanced model atmospheres and the sample standard deviation for the latter. Also listed are differential abundance estimates for HD 135485 taken from Trundle et al. (2001). The differential abundances are defined as $\left[\frac{X}{H}\right]=\log \epsilon(X)_{\text {star }}-\log \epsilon(X)_{\text {standard }}$.

\begin{tabular}{|c|c|c|c|c|c|c|c|c|c|c|c|}
\hline \multirow[t]{3}{*}{ Ion } & \multicolumn{4}{|c|}{$279-08$} & \multicolumn{4}{|c|}{ 393-03 } & \multicolumn{3}{|c|}{ HD 135485} \\
\hline & \multirow[t]{2}{*}{ No. } & \multicolumn{2}{|c|}{$\left[\frac{X}{H}\right]$} & \multirow[t]{2}{*}{$\sigma$} & \multirow[t]{2}{*}{ No. } & \multicolumn{2}{|c|}{$\left[\frac{X}{H}\right]$} & \multirow[t]{2}{*}{$\sigma$} & \multirow[t]{2}{*}{ No. } & \multirow[t]{2}{*}[\frac{X}{H}]{} & \multirow[t]{2}{*}{$\sigma$} \\
\hline & & Normal & Enhanced & & & Normal & Enhanced & & & & \\
\hline He I & 4 & 0.31 & 0.28 & 0.07 & 3 & 0.05 & 0.02 & 0.20 & 4 & 0.31 & 0.06 \\
\hline $\mathrm{C}_{\mathrm{II}}$ & 5 & 0.71 & 0.68 & 0.10 & 3 & 0.56 & 0.55 & 0.29 & 3 & 0.66 & 0.30 \\
\hline N II & 5 & 1.08 & 0.94 & 0.14 & 11 & 1.03 & 1.06 & 0.10 & 7 & 1.37 & 0.14 \\
\hline O II & 11 & 0.44 & 0.29 & 0.23 & 16 & 0.29 & 0.34 & 0.20 & 9 & 0.45 & 0.19 \\
\hline Mg II & 1 & -0.07 & -0.03 & - & 1 & 0.15 & 0.07 & - & 5 & 0.62 & 0.06 \\
\hline Al III & 2 & 0.47 & 0.36 & 0.37 & 2 & 0.61 & 0.62 & 0.36 & 3 & 0.68 & 0.05 \\
\hline Si II/III & 5 & 0.08 & 0.11 & 0.17 & 4 & 0.22 & 0.20 & 0.12 & 7 & 0.52 & 0.05 \\
\hline S II & 6 & 0.31 & 0.34 & 0.13 & 8 & 0.42 & 0.38 & 0.17 & 19 & 0.56 & 0.11 \\
\hline Fe III & - & - & - & - & 2 & 0.38 & 0.40 & 0.06 & 3 & 0.49 & 0.05 \\
\hline
\end{tabular}

M 13. They found that the abundances of many elements differed from those implied by the underlying metallicity of the globular cluster, which they attributed to diffusion. However the $\mathrm{Mg}$ abundances in all their targets were consistent with the underlying cluster metallicity and they concluded that this element was not significantly affected by diffusion. Subsequently, Moehler et al. (2000) deduced Mg abundances for 19 stars in the globular cluster NGC6752 and again found them tightly correlated with the cluster's metallicity.

Assuming that the $\mathrm{Mg}$ abundance is a good indicator of the underlying metallicity of our targets, it would appear that both stars formed from material with a relatively normal (i.e. young B-type stellar) composition. The overabundances seen in the abundances of $\mathrm{Al}, \mathrm{Si}, \mathrm{S}$ and $\mathrm{Fe}$ could then reflect the effects of diffusion processes as Behr et al. found significant enhancements in both the $\mathrm{Si}$ and $\mathrm{Fe}$ abundances in their targets. The explanation for the CNO elements is not so clear cut as both the effects of diffusion and nucleosynthetic contamination may be important. However if the surface contamination was from material processed by the CNO bi-cycle, then modest depletions of $\mathrm{C}$ and $\mathrm{O}$ would be expected. Hence the observed enhancements of these elements is probably due to diffusion. The significantly larger enhancement of nitrogen may at least be partly due to nucleosynthetically processed material.

Behr et al. (1999) deduced significant enhancements of both $\mathrm{P}$ and $\mathrm{Cr}$ in their targets, which they attributed to the effects of diffusion. A careful investigation of the spectra of our targets indicated that there was no evidence for lines due to P II. The situation for $\mathrm{Cr}$ was more ambiguous with possible identifications for some $\mathrm{Cr}$ II features. However other lines observed by, for example, Trundle et al. (2001) could not be identified. Hence for both species we adopted a conservative approach and set limits on the equivalent widths that would have been observable with corresponding limits on the element abundances (summarised in Table 3). For Cr, the limits are not particularly stringent with for example Venn (1995) finding a $\mathrm{Cr}$ abundance of approximately 5.6 dex from A-type supergiants. Hence a $\mathrm{Cr}$ enhancement of approximately a factor ten could be present in 
Table 5. Differential abundance analysis for the broad lined stars 456-39 and 591-05. The format is similar to that for Table 4. Also included are results for LS5130, a star previously analysed by Smartt et al. (2001) using similar methods.

\begin{tabular}{|c|c|c|c|c|c|c|c|c|c|c|c|}
\hline \multirow[t]{3}{*}{ Ion } & \multicolumn{4}{|c|}{$456-39$} & \multicolumn{4}{|c|}{ 591-05 } & \multicolumn{3}{|c|}{ LS 5130} \\
\hline & \multirow[t]{2}{*}{ No. } & \multicolumn{2}{|c|}{$\left[\frac{X}{H}\right]$} & \multirow[t]{2}{*}{$\sigma$} & \multirow[t]{2}{*}{ No. } & \multicolumn{2}{|c|}{$[\underline{X}]$} & \multirow[t]{2}{*}{$\sigma$} & \multirow[t]{2}{*}{ No. } & \multirow[t]{2}{*}[\frac{X}{H}]{} & \multirow[t]{2}{*}{$\sigma$} \\
\hline & & Normal & Enhanced & & & Normal & Enhanced & & & & \\
\hline $\mathrm{He} \mathrm{I}$ & 2 & 0.09 & 0.07 & 0.15 & 3 & 0.12 & 0.13 & 0.22 & - & - & \\
\hline C II & 3 & 0.51 & 0.47 & 0.12 & 1 & 0.71 & 0.74 & - & 9 & 0.23 & 0.15 \\
\hline N II & 8 & 0.27 & 0.24 & 0.13 & 1 & 0.33 & 0.39 & - & 19 & 0.15 & 0.13 \\
\hline O II & 26 & 0.12 & 0.08 & 0.13 & 11 & 0.10 & 0.10 & 0.21 & 35 & -0.06 & 0.16 \\
\hline Mg II & 1 & 0.19 & 0.15 & - & 1 & 0.15 & 0.19 & - & 1 & 0.42 & - \\
\hline Si II/III/IV & 3 & 0.21 & 0.16 & 0.07 & 2 & 0.20 & 0.26 & 0.01 & 9 & 0.35 & 0.13 \\
\hline $\mathrm{Fe}$ III & 1 & 0.42 & 0.39 & - & - & - & - & - & 3 & 0.13 & 0.18 \\
\hline
\end{tabular}

our targets but undetected. By contrast the P abundance limits, indicate that abundances for this element in not significantly enhanced over that found in normal B-type stars (see, for example, Trundle et al. 2001).

Although we believe that these two stars are probably evolved objects similar to HD 135485, there atmospheric parameters are similar to those of young main sequence objects. This can be seen in Fig. 2, where the targets lie mear to the zeroage main sequence tracks (although their gravities are slightly higher than predicted by such models). This have implications for the use of early-type stars as tracers of the chemical composition of the interstellar medium. In particularly evolved objects may be misidentified as young (near) main sequence objects. However evolved objects are normally slowly rotating (and hence have very sharp lined spectra), whilst their chemical composition are often affected diffusion and dredge-up of nucleosynthetically processed material. Hence normally it should be possible to identify such objects.

\subsection{Broad-lined stars - 456-39 and 591-05}

Analyses of the two broad-lined stars were more problematic than those of the narrow-lined stars discussed above. This was due to the difficulty of measuring the shallow absorption features present in their spectra. However it was still possible to estimate absolute abundances for $\mathrm{C}, \mathrm{N}, \mathrm{O}, \mathrm{Mg}, \mathrm{Si}$ and for one star Fe. In general these absolute abundances imply a modest enhancement for all elements, although the $\mathrm{O}$ abundances appear to be near normal. Additionally for both stars there is no evidence for a relative enrichment in nitrogen, which implies that significant rotational mixing of material from the stellar core has not occurred in this case.

For the differential abundance analysis of the target star 456-39, the standard star, HR 2387 was employed. 456-39 has only a moderate projected rotational velocity $\left(45 \mathrm{~km} \mathrm{~s}^{-1}\right)$ and hence it was possible to measure a significant number of absorption lines down to an equivalent widths limit of approximately $40 \mathrm{~m} \AA$. As found in the absolute abundance analysis, a general metal enhancement is implied for this star, ranging from $0.12 \mathrm{dex}$ for $\mathrm{O}$ to $0.51 \mathrm{dex}$ for $\mathrm{C}$. The He abundance, which was deduced from non-diffuse lines, may also show a marginal enhancement but this does not appear to be statistically significant. Although the Fe abundance is based on a
Table 6. Distances were estimated from UBV measurements and by employing the same method as that used by Smartt et al. (2001). A distance estimate is also given for the lower mass cases of the three sub-luminous stars.

\begin{tabular}{lll}
\hline \hline Star & $\begin{array}{l}d \text { (high mass) } \\
(\mathrm{kpc})\end{array}$ & $\begin{array}{l}d \text { (low mass) } \\
(\mathrm{kpc})\end{array}$ \\
\hline $279-08$ & 3.7 & 1.0 \\
$279-19$ & 15 & 3.6 \\
$393-03$ & 4.2 & 1.1 \\
$456-39$ & 3.2 & - \\
$591-05$ & 11 & - \\
\hline
\end{tabular}

single line, it is interesting that it yields a similar overabundance to other elements.

The results for the target star 591-05 are less reliable due to its larger projected rotational velocity (see Table 1), which limited equivalent width measurements to lines that were stronger than $80 \mathrm{~m} \AA$. The standard star HR 5595 was used for comparison in the differential abundance analysis. Helium again may be slightly enhanced although this result is not statistically significant. The metal abundances are based on small numbers of absorption features but show a similar pattern to that for 456-09, with the $\mathrm{C}$ overabundance being the highest while the $\mathrm{O}$ abundance is near normal.

Smartt et al. (2001) have analysed the spectrum of four B-type targets towards the Galactic Centre, which they believe to be young unevolved stars; the methods used were very similar to those discussed here. Their best observed target was LS 5130 due to the high $S / N$ ratio of its spectrum and its low projected rotational velocity. In Table 5, the results for LS 5130 are summarised and show a similar pattern to 45609 and 591-05. In particular, there is a general enhancement of metals apart from the $\mathrm{O}$ abundance that appears to be near normal.

The targets studied by Smartt et al. had $V$-magnitudes in the range 11.1-12.1 and were estimated to lie at distances ranging from 4 to $9 \mathrm{kpc}$ from the Sun. Because of their different Galactic longitudes, their estimated distances from the Galactic Centre were between 2 and $5 \mathrm{kpc}$. Hence as discussed by Smartt et al., they may provide probes of the composition 
of the interstellar medium at approximately $4 \mathrm{kpc}$ from the Galactic Centre. Our two targets are approximately one magnitude fainter (see Table 1). Distance estimates for all five of our targets were obtained by employing the same method as that used by Smartt et al. As can be seen in Table 6, this led to estimates of $3.2 \mathrm{kpc}$ for 456-39 and $11 \mathrm{kpc}$ for 591-05. In turn adopting a distance to the Galactic Centre of $8.5 \mathrm{kpc}$, we can estimate their distances from the Galactic Centre. The galactic longitude of 456-39 is effectively zero (see Dufton et al. 1999, 2001), implying a galactocentric distance of $5.3 \mathrm{kpc}$. The longitude of 591-05 is $12^{\circ}$ leading to a distance from the Galactic Centre of $3.2 \mathrm{kpc}$.

The distance estimates to our targets will be affected by many uncertainties including errors in the atmospheric parameters and the interstellar extinction. However, they lead to galactocentric distances that are similar to those found by Smartt et al. for their four targets. Additionally the chemical composition of stars within and between the two groups show similarities indicating that they may have originated from comparable interstellar material. Hence in Table 8, we summarise the range of elemental abundances found for all six targets. Additionally, we list the mean abundance and standard deviation (weighted by the number of lines included in the analysis for each star) for each element; we note that these results would be effectively unchanged if the results for each star were weighted equally.

The range of abundances estimates found for each ion indicate a general enhancement of metals. Indeed for all elements apart from oxygen, the mean abundance estimates would imply that this is typically 0.4 dex. Given the difference between the galactocentric distance of the sun and this group of stars of approximately $5 \mathrm{kpc}$, this would imply linear logarithmic

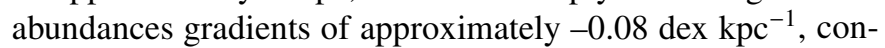
sistent with that found previously in the anticentre direction from observations of stars, $\mathrm{H}$ II regions and PN nebula (see, for example, Rolleston et al. (2000) and references therein). The standard deviations for these abundance estimates are relatively large (typically $\pm 0.20 \mathrm{dex}$ ), which will be in part due to uncertainties in the analysis. However they may also reflect real differences in the metal abundances in individual stars. This would be consistent with the stars being formed from material with a range of galactocentric distances as implied by their current galactocentric distance estimates ranging from 2 to $6 \mathrm{kpc}$ (also as discussed by Smartt et al. (2001), these targets lie at some distance from the galactic plane and may have been born at a different galactocentric distance to that currently observed).

For oxygen, the situation appears to be radically different with a mean abundance estimate that is effectively normal. Additionally both the range in abundance estimates and the standard deviation of the mean are far smaller than for the other elements. This may now reflect the uncertainties in the estimates without there being any additional contributions from variations in the stellar oxygen abundances. Hence we confirm the findings of Smartt et al. (2001) that stellar observations imply that the oxygen abundance gradient flattens at small galactocentric distances. This is consistent with the small abundance gradients for oxygen found in M 31 by Trundle et al. (2002) from observations of early-type supergiants. However it should be noted that their results covered a relatively wide range of
Table 7. Differential abundance analysis for the low gravity star 27919. An enhanced metallicity analysis was not necessary for this star.

\begin{tabular}{lrrr}
\hline \hline Ion & $\begin{array}{r}\text { No. of } \\
\text { lines }\end{array}$ & $\begin{array}{r}{\left[\frac{X}{H}\right.} \\
\text { Normal }\end{array}$ & $\sigma$ \\
\hline He I & 3 & +0.13 & 0.15 \\
C II & 2 & -0.22 & 0.40 \\
N II & 4 & -0.02 & 0.36 \\
O II & 27 & +0.01 & 0.30 \\
Si III/IV & 4 & -0.35 & 0.17 \\
Fe III & 1 & +0.15 & 0.00 \\
\hline
\end{tabular}

galactocentric distances (5-20 kpc) and that they also deduced small gradients for magnesium and silicon. Hence the agreement for the oxygen abundance gradients in the two galaxies may be fortuitious and indeed reflect independent phenomena. Afflerbach et al. (1997) and Rudolph et al. (1997) have estimated abundances for nitrogen, oxygen and sulphur in $\mathrm{H}$ II regions from infra-red emission lines. Although Rudolph et al. were not able to independently determine the oxygen and sulphur abundances, both studies found significant abundance gradients (typically -0.06 to $-0.10 \mathrm{dex} \mathrm{kpc}^{-1}$ ) with no evidence for any flattening of the gradient at low galactocentric distance.

The apparent flattening of the oxygen abundance gradient in the inner Galaxy is a puzzling phenomenon. As discussed briefly in Smartt et al. (2001) there is limited quantitative modelling of the chemical evolution of metal rich galactic regions combined with detailed metallicity dependent yields. This precludes comparison of our results with model predictions on an individual element basis. For example barred galaxies tend to have shallower abundance gradients than non-barred ones (see Martin \& Roy 1994 and references therein), with the explanation being that gas flows along the bar induce strong mixing and reduce the gradients. If the Milky Way is indeed a barred galaxy (Weinberg 1992) then this could be a explanation for the decreasing $\mathrm{O}$ gradient. But that would not explain why the $\mathrm{Mg}$ and $\mathrm{Si}$ abundances continue to rise significantly. Both $\mathrm{Mg}$ and $\mathrm{Si}$ are produced along with $\mathrm{O}$ in supernovae of type II, and with similar production sites it is hard to envisage gas flows along the bar affecting one of the elements but not the others. In addition, the models of Portinari \& Chiosi $(1999,2000)$ also indicate that the $\mathrm{O}$ abundance gradient may indeed flatten off in the region $3 \lesssim R_{\mathrm{g}} \lesssim 6 \mathrm{kpc}$, but again their models also predict the same should occur for the type II supernovae products $\mathrm{Mg}$ and $\mathrm{Si}$. There has been some work into metallicity dependent yields from massive stars and in particular the yield from corecollapse supernovae at varying metallicities. E.g. Prantzos et al. (1994), Maeder (1992). In these studies there is some suggestion that the $\mathrm{O}$ yields from type II supernovae do decrease as metallicity increases, however there is no detailed study of all the $\alpha$-processed elements, that would allow quantitative comparison with our results. 
Table 8. Range of abundances estimates for different elements for the normal young targets observed here and by Smartt et al. (2001). Also listed are the mean values and standard deviations.

\begin{tabular}{lrr}
\hline \hline Element & Range & Mean \\
\hline $\mathrm{C}$ & $+0.1 \rightarrow+0.7$ & $+0.34 \pm 0.25$ \\
$\mathrm{~N}$ & $+0.2 \rightarrow+0.6$ & $+0.33 \pm 0.20$ \\
$\mathrm{O}$ & $-0.1 \rightarrow+0.2$ & $+0.06 \pm 0.11$ \\
$\mathrm{Mg}$ & $+0.1 \rightarrow+0.7$ & $+0.37 \pm 0.20$ \\
$\mathrm{Si}$ & $+0.2 \rightarrow+0.8$ & $+0.39 \pm 0.22$ \\
$\mathrm{~S}$ & $+0.1 \rightarrow+0.7$ & $+0.39 \pm 0.17$ \\
\hline
\end{tabular}

\subsection{Low gravity star $-279-19$}

The star 279-19 differed from our other targets in that it had a significantly lower surface gravity and appeared to have a near normal metallicity; hence an enhanced metallicity analysis was not considered necessary. The differential analysis was relative to a star in the double cluster $\mathrm{h}$ and $\chi$ Persei, $\mathrm{BD}+56^{\circ} 502$, which has been classified as a giant. Vrancken et al. (2000) undertook a non-LTE analysis of its spectrum and found no evidence for any mixing of nucleosynthetically processed material to its surface. Hence we believe that it should provide a reliable standard.

The chemical composition of 279-19 appears relatively normal particularly considering the uncertainties in the individual abundance estimates. Its atmospheric parameters are consistent with it being either a normal young star or an evolved low mass object. If it is the former, a bolometric magnitude of -7.31 leads to a distance estimate of $15 \mathrm{kpc}$ from the Sun and approximately $3 \mathrm{kpc}$ from the galactic plane. Although this is possible, we believe that it is more likely that 279-19 is an evolved star. Then one possibility is that it is at a postasymptotic-giant branch evolutionary stage - see, for example, Blöcker \& Schönberner (1990). The evolutionary models of Schönberner $(1983,1987)$ would imply that $279-19$ had a mass of approximately 0.56 solar masses and was evolving relatively slowly from the asymptotic-giant branch to become a white dwarf.

Indirect evidence for this evolutionary scenario is that the $\mathrm{H} \alpha$ line is observed to be in emission (see Fig. 3). Additionally the spatial extent of the emission is significantly larger than that of the stellar continuum. Indeed after allowing for effects of seeing (with a $F W H M \simeq 1.5 \operatorname{arcsec}$ ), the $F W H M$ of the spatial extent of the nebular emission is approximately 4.6 arcsec, which would correspond to approximately 0.080 parsecs for a stellar distance of $3.6 \mathrm{kpc}$. For LSIV -12 111, Conlon et al. (1993) deduced an angular extent for the nebular emission of 1.2 arcsec corresponding to a spatial extent of 0.023 parsec for their distance estimate. Hence the spatial extent of the nebular emission in 279-19 would appear to be a factor of 3-4 larger than that for LSIV -12111. We note that the estimated effective temperature of 279-19 is larger than that for LSIV -12 111 (Ryans et al. 2003) and this may explain at least in part the different sizes of the surrounding nebulae.

Alternatively 279-19 could have evolved from the extended horizontal branch. Dorman et al. (1993) have discussed this

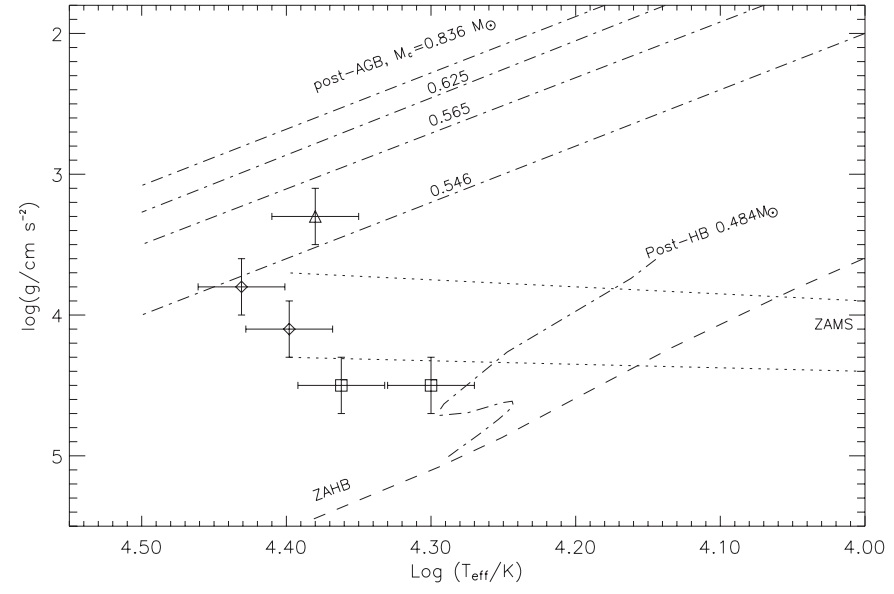

Fig. 2. The position of our targets in an effective temperature versus gravity diagram. The narrow-lined stars are represented by squares, the broad-lined stars by diamonds and the low gravity star by a triangle. Representative error bars of $\pm 2000 \mathrm{~K}$ in effective temperature and \pm 0.2 dex in logarithmic gravity are adopted. Also shown are the theoretical positions of the zero-age hydrogen burning main- sequence (ZAMS), zero-age horizontal branch (ZAHB), post-horizontal branch (post-HB) and post-asymptotic giant branch (post-AGB) (Bertelli et al. 1994; Sweigart 1987; Dorman et al. 1993; Schönberner 1993 respectively).

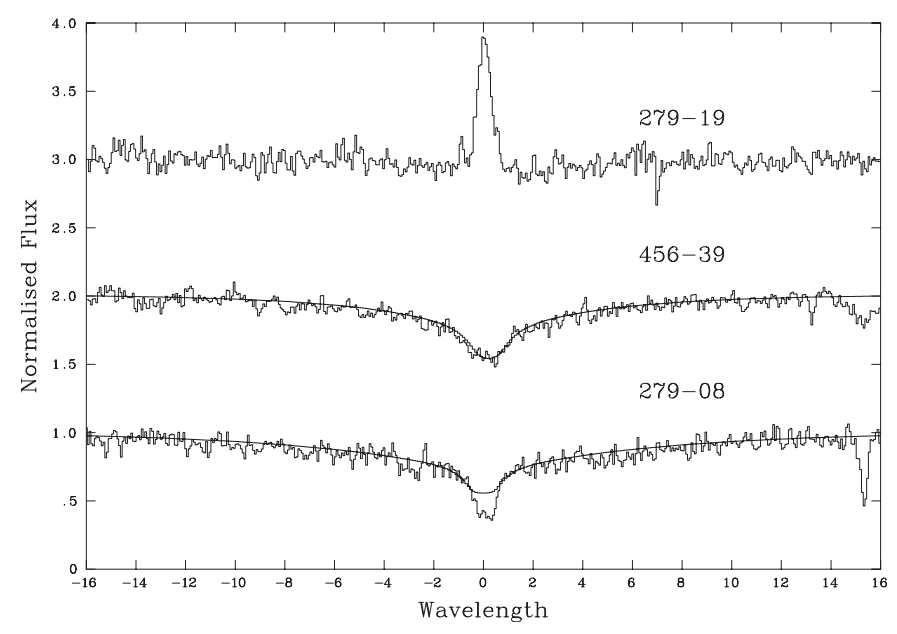

Fig. 3. Spectra of the $\mathrm{H} \alpha$ region for three of our targets. The pure absorption spectra of 279-08 and 456-39 are consistent with them being on the blue horizontal branch and the hydrogen burning main sequence respectively. The strong emission for 279-19 is consistent with it being a post-asymptotic-branch object.

scenario and in particular find that if the star retains sufficient envelope mass it will start to ascend the AGB but will evolve off it prior to the onset of a thermal pulse. Dorman et al. characterise such objects as post-early AGB stars and find they are generally less luminous $\left(\log L / L_{\odot} \leq 3\right)$ than PAGB stars. From our atmospheric parameters and inferred masses, the luminosity of $279-19$ is approximately $2 \times 10^{3} L_{\odot}$, which appears to be inconsistent with this scenario. Hence we conclude that $279-19$ is probably a PAGB star but other possibilities cannot be definitely ruled out. Then its chemical composition would be consistent with that found for other PAGB candidates, such as LSIV-12 111 (Ryans et al. 2003), 
although it does not show the marked $\mathrm{C}$ deficiency that has been found in some targets with similar atmospheric parameters e.g. PHL 1580 (McCausland et al. 1992)

\section{Conclusions}

The principle aim of the survey of blue objects towards the Galactic Centre reported by Dufton et al. $(1999,2001)$ was to identify young early-type stars. The high extinction towards the Galactic Centre meant that the survey concentrated on regions with galactic latitudes between 5 and $10^{\circ}$ from the Galactic plane. From a combination of photographic photometry and low dispersion multi-object spectroscopy, Dufton et al. identified approximately 130 objects in ten Schmidt plate fields which appeared to be B-type objects. Of these, 32 objects were identified as having a spectral type of B3 or earlier and hence particularly suitable for abundance studies as their spectra would contain features due to $\mathrm{C}, \mathrm{N}, \mathrm{O}, \mathrm{Mg}, \mathrm{Si}$ and possibly Fe.

The high resolution spectroscopy presented here had two main aims. Firstly to validate the selection procedure used by Dufton et al. and then to use the spectra to investigate the chemical composition towards the Galactic Centre. Encouragingly all thirteen targets appeared to be early B-type stars. However for most of them the large projected rotational velocity coupled with their moderate spectral $S / N$ ratios precluded a model atmosphere analysis. Of equal concern is that of the five remaining targets only two could be confidently identified as young objects. Hence it would appear that an additional filtering of the targets identified by Dufton et al. is required. This would ideally be achieved using moderate dispersion spectroscopy (with a velocity resolution of approximately $20-40 \mathrm{~km} \mathrm{~s}^{-1}$ ), which should be sufficient to exclude both fast rotators and very narrow-lined evolved objects. Additionally such spectroscopy should allow stellar atmospheric parameters to be deduced from the hydrogen and helium features (see Dufton et al. 1999). In turn this would allow low-gravity objects, which may be PAGB stars, to be excluded, which is important as such objects can show significant broadening (probably due to macroturbulence) in their spectra (see, for example, Ryans et al. 2003) and hence the use of line widths alone is not sufficient to identify these objects.

For the specific objects analysed here, two appear to be evolved blue horizontal branch objects with an initially near normal metallicity whose surface composition has subsequently been modified by diffusion and possibly contamination by nucleosynthetically processed material. A third object could be a normal early-type supergiant but is more likely to be an evolved object evolving from the AGB. The remaining two objects are probably young objects and show enhanced abundances of $\mathrm{N}, \mathrm{C}, \mathrm{Mg}$ and $\mathrm{Si}$, consistent with their formation in the inner part of the Galaxy. However their $\mathrm{O}$ abundances are normal, confirming results found previously for other stars in this region by Smartt et al. (2001). This would in turn imply a flat abundance gradient for this element in the inner region of our Galaxy.

Acknowledgements. K.E.M. and S.J.S. acknowledge financial support from the NI Department of Education and Learning and PPARC respectively. We are very grateful to Dave Kilkenny for providing us with $U B V$ photometry for some of our targets. Spectroscopic data were obtained at the Anglo-Australian Observatory in Siding Spring, New South Wales and photometric data were obtained at the $1.0 \mathrm{~m}$ at the South African Astronomical Observatory.

\section{References}

Afflerbach, A., Churchwell, E., \& Werner, M. W. 1997, ApJ, 478, 190 Behr, B. B., Cohen, J. G., McCarthy, J. K., \& Djorgovski, S. G. 1999, ApJ, 517, L135

Bertelli, G., Bressan, A., Chiosi, C., Fagotto, F., Nasi, E. 1994, A\&AS, 106, 275B

Blöcker, T., \& Schönberner, D. 1990, A\&A, 240, L11

Bresolin, F., Kudritzki, R. P., Lennon, D. J., et al. 2002, ApJ, 580, 213

Conlon, E. S., Dufton, P. L., McCausland, R. J. H., Keenan, F. P. 1993, ApJ, 408, 593

Daflon, S., \& Cunha, K., 2003, in IAU Symp. 212, ed. van der Hucht, D., Herrero, A. \& Estaban, C. 162

Dorman, B., Rood, R. T., \& O’Connell, R. W. 1993, ApJ, 419, 596

Dufton, P. L. 1998, Proc. of the BMW-II conference, ASP Conf. Ser., 169

Dufton, P. L., Smartt, S. J., \& Hambly, N. C. 1999, A\&AS, 139, 231

Dufton, P. L., Smartt, S. J., \& Hambly, N. C. 1999, A\&AS, 373, 608

Gies, D. R., \& Lambert, D. L. 1992, ApJ, 387, 673

Gummersbach, C. A., Kaufer, A., Schäfer, D. R., Szeifert, T., \& Wolf B. 1998, A\&A, 342, 426

Hambly, N. C., Rolleston, W. R. J., Keenan, F. P., Dufton, P. L., \& Saffer, R. A. 1997, ApJS, 111, 419

Howarth, I. D., Murray, M. J., \& Mills, D. 1994, Starlink User Note, No. 50

Howarth, I. D. 1998, Proc. of the BMW-II conference, ed. I. D. Howarth, ASP Conf. Ser., 169

Hubeny, I. 1988, Comp. Phys. Comm., 52, 103

Hubeny, I., Lanz, T. 1995, ApJ, 439, 875

Jeffery, C. S. 1991, Newsletter on Analysis of Astronomical Spectra, No. 16,17

Kilian, J. 1992, A\&A, 262, 171

Korn, A. J., Keller, S. C., Kaufer, A., et al. 2002, A\&A, 385, 143

Lennon, D. J., Dufton, P. L., Keenan, F. P., \& Holmgren, D. E. 1991, A\&A, 252, 498

Maeder, A. 1992, A\&A, 264, 105

Martin, P., \& Roy, J.-R. 1994, 424, 599

Massey, P. 1997, A User's Guide to CCD reductions with IRAF, NOAO Laboratory

Mathys, G., Andrievsky, S. M., Barbuy, B., Cunha, K., \& Korotin, S. A. 2002, A\&A, 387, 890

McCausland, R. J. H., Conlon, E. S., Dufton, P. L., \& Keenan, F. P. 1992, ApJ, 394, 298

Mihalas, D. 1972 ApJ, 176, 139

Moehler, S., Sweigert, A. V., Landsmann, W. B., \& Heber, U. 2000, A\&A, 360, 120

Monteverde, M. I., Herrero, A., Lennon, D. J., \& Kudritzki, R. P. 1997, ApJ, 474, L107

Portinari, L., \& Chiosi, C. 1999, A\&A, 350, 827

Portinari, L., \& Chiosi, C. 2000, A\&A, 355, 929

Prantzos, N., Vangioni-Flam, E., \& Chauveau, S. 1994, A\&A, 285, 132

Reed, B. C. 1993, ApJS, 87, 367

Reed, B. C., \& Beatty, A. E. 1995, ApJS, 97, 189

Rolleston, W. R. J., Brown, P. J. F., Dufton, P. L., \& Howarth, I. D. 1996, A\&A, 315, 95 
Rolleston, W. R. J., Dufton, P. L., McErlean, N. D., \& Venn, K. A. 1999, A\&A, 348, 728

Rolleston, W. R. J., Smartt, S. J., Dufton, P. L., \& Ryans, R. S. I. 2000, A\&A, 363, 537

Rolleston, W. J. R., Venn, K. A., Tolstoy, E., \& Dufton, P. L. 2003, A\&A, 400, 21

Rudolph, A. L., Simpson, J. P., Haas, M. R., Erickson, E. F., \& Fich, M. 1997, ApJ, 489, 94

Ryans, R. S. I., Dufton, P. L., Keenan, F. P., et al. 1997, ApJ, 490, 267

Ryans, R. S. I., Dufton, P. L., Mooney, C. J., et al. 2003, A\&A, 401, 1119

Schlegel, D. J., Finkbeiner, D. P., \& Davis, M. 1998, ApJ, 500, 525S

Schönberner, D. 1983, ApJ, 272, 708

Schönberner, D. 1987, in Late Stages of Stellar Evolution, ed. S. Kwok, \& S. R. Pottasch (Dordrecht, Reidel), 359

Schonberner D. 1993, in Planetary Nebulae ed. Weinberger R., \& Acker A., IAU Symp. 155, 415

Smartt, S. J., \& Rolleston, W. R. J. 1997, ApJ, 481, L47

Smartt, S. J., Dufton, P. L., \& Rolleston, W. R. J. 1996a, A\&AS, 116, 483
Smartt, S. J., Dufton, P. L., \& Rolleston, W. R. J. 1996b, A\&A, 310, 123

Smartt S. J., Venn, K. A, et al. 2001, A\&A, 367, 86

Smartt, S. J., Crowther, P. A., Dufton, P. L., Lennon, D. J., et al. 2001, MNRAS, 325, 257

Sweigart, A. V. 1987, ApJS, 65, 95

Tody, D. 1986, IRAF User Manual, NOAO Laboratory

Trundle, C., Dufton, P. L., Rolleston, W. R. J., et al. 2001, MNRAS, 328, 291

Trundle, C., Dufton, P. L., Lennon, D. J., Smartt, S. J., \& Urbaneja, M. A. 2002, A\&A, 395, 519

Venn, K. A, Smartt, S. J., Lennon, D. J., \& Dufton, P. L. 1998, A\&A, 334, 987

Venn, K. A. 1995, ApJS, 99, 659

Venn, K. A., Lennon, D. J., Kaufer, A., et al. 2001, ApJ, 547, 765

Vrancken, M., Lennon, D. J., Dufton, P. L., \& Lambert, D. L. 2000, A\&A, 358, 639

Weinberg, M. D. 1992, ApJ, 384, 81

Willmarth, D., \& Barnes, J. 1994, A User's Guide to Reducing Echelle Spectra with IRAF, NOAO Laboratory 